\title{
Incomplete Staging (pM)
}

National Cancer Institute

\section{Source}

National Cancer Institute. Incomplete Staging (pM). NCI Thesaurus. Code C159724.

A lack of clinical and/or pathology information to assign a pM stage conclusively. 